\title{
Evaluation of Effectiveness of Photodynamic Therapy With Low-level Diode Laser in Nonsurgical Treatment of Peri-implantitis
}

\author{
Ehsan Birang ${ }^{1}$, Mohammad Reza Talebi Ardekani ${ }^{2}$, Mahboobeh Rajabzadeh ${ }^{3 *}$, Gloria Sarmadi ${ }^{4}$, Reza Birang ${ }^{3}$ \\ Norbert Gutknecht ${ }^{5}$ \\ ${ }^{1}$ School of Dentistry, Shahid Beheshti University of Medical Sciences, Tehran, Iran \\ ${ }^{2}$ Department of Periodontics, Shahid Beheshti University of Medical Sciences, Tehran, Iran \\ ${ }^{3}$ Torabinejad Dental Research Center, Department of Periodontics, School of dentistry, Isfahan University of Medical \\ Sciences, Isfahan, Iran \\ ${ }^{4}$ Torabinejad Dental Research Center, Department of Oral and Maxillofacial Radiology, School of Dentistry, Isfahan \\ University of Medical Sciences, Isfahan, Iran \\ ${ }^{5}$ Department of Conservative Dentistry, Rwth Hospital, Aachen, Germany
}

*Correspondence to

Mahboobeh Rajabzadeh, DDS;

Torabinejad Dental Research Center Department of Periodontics, School of Dentistry, Isfahan University of Medical Sciences, Isfahan, Iran.

Tel: +98-9155013649;

Fax:+98-3136687080;

Email:

mahboobe_6630@yahoo.com

Published online 27 June 2017

\begin{abstract}
Introduction: Side effects related to antibiotic therapy for peri-implantitis are rare in laser therapy (LT); therefore, the aim of this study was to evaluate the effectiveness of LT and photodynamic therapy (PDT) on patients with primary peri-implantitis.

Methods: In this randomized clinical trial, 40 implants presenting primary peri-implantitis in 20 patients with a mean age of 52.6 years old were included using the simple sampling technique. Periodontal treatment comprising scaling and root planing (SRP) was accomplished for the whole mouth while mechanical debridement with titanium curettes and air polishing with sodium bicarbonate powder was accomplished around the implants. The implants were randomly divided into two groups and treated with LT (control) and PDT (test). The clinical indices were measured at baseline, 6 weeks and 3 months after treatment. Real-time polymerase chain reaction (PCR) was used for analysis of microbial samples at baseline and 3-month follow-up. Data were analyzed with SPSS 20, using repeated-measures analysis of variance (ANOVA) and Friedman's and MannWhitney tests $(\alpha=0.05)$.

Results: Both groups showed statistically significant improvements in terms of bleeding on probing $(P<0.001)$, probing pocket depth (PPD) $(P=0.006)$ and modified plaque index $(P<0.001)$, with no significant differences between the 2 groups $(P>0.05)$. The number of Aggregatibacter actinomycetemcomitans $(P=0.022)$, Tannerella forsythia $(P=0.038)$ and Porphyromonas gingivalis $(P=0.05)$ in the test group and Porphyromonas gingivalis $(P=0.015)$ in the control group significantly decreased.

Conclusion: The results suggested that LT and PDT have significant short-term benefits in the treatment of primary peri-implantitis.

Keywords: Laser; Photosensitizer; Photodynamic therapy.
\end{abstract}

\section{Introduction}

Peri-implantitis is defined as the inflammation of supporting tissues of dental implants in association with bone loss, which will result in the progressive destruction of bone around the implant, if left untreated. ${ }^{1}$ Peri-implantitis is treated through mechanical and chemical techniques. Usually, mechanical techniques alone cannot eliminate microorganisms from the major parts of pockets around the implants. In addition, after mechanical debridement with carbon fiber curettes alone, without any adjunctive treatment, pocket depth improves but not significantly. ${ }^{2}$ In addition, various systemic and local chemical antimicrobial agents have been introduced for the treatment of peri-implantitis, which suppress periodontopathogens more effectively compared to mechanical techniques, and improve the results of conventional mechanical therapeutic techniques. ${ }^{3-7}$ Some disadvantages of antimicrobial agents' use, (such as antibiotics) include an increase in the counts of bacteria resistant to these agents, the need for the use of different antibiotics due to the diversity of periodontopathogens, an increase in the number of immunosuppressed patients and the incidence of unfavorable reactions. Considering the complications above, it is necessary to expand research

Please cite this article as follows: Birang R, Shahabooei M, Rajabzadeh M, Sarmadi G, Birang E. Evaluation of effectiveness of photodynamic therapy with low-level diode laser in nonsurgical treatment of peri-implantitis. J Lasers Med Sci. 2017;8(3):136-142. doi:10.15171/jlms.2017.25. 
in an attempt to find alternative antimicrobial techniques. One of them is the application of lasers and photodynamic therapy (PDT), which might be effective in eliminating microbes in local and superficial infections. ${ }^{8-11}$

Nowadays, treatments with laser alone or PDT in association with photo-sensitizers have become popular as new therapeutic techniques in dentistry, and are used in a large number of dental procedures, including treatment of periodontal diseases, ${ }^{12}$ peri-implant infections ${ }^{13,14}$ and endodontic infections. ${ }^{15}$ Given the non-invasive and local nature of lasers, the incidence of many side effects associated with the use of antibiotics, including injuries to the gastrointestinal mucosa and occurrence of drug allergies, are improbable with the use of lasers. In addition, since the photodynamic technique exerts its bactericidal effect through free oxygen species and hydroxyl radials, it appears that it is rare for resistance to occur against PDT. ${ }^{16-19}$ A review study evaluated the results of several studies in an attempt to evaluate the effect of PDT on periodontal diseases, concluding that the use of photosensitizing dyes followed by their activation with visible light can effectively destroy periodontopathogens. ${ }^{20}$

Of all the lasers available, diode laser is used for the debridement of periodontal pockets and removal of the epithelial lining, including the granulomatous tissues. $^{21,22}$ This laser has a superb hemostatic effect and can be applied for cutting and coagulating gingiva and mucosa. ${ }^{23}$ In addition, this laser can contact the implant surface without melting, cracking or making it concave. ${ }^{1}$ Therefore, the aim of the present study was to evaluate the clinical and microbiological effects of the use of EmunDo dye as a photosensitizer, in association with the applications of 810-nm diode laser beams and to compare it with the application of laser alone for the treatment of peri-implantitis.

\section{Methods}

Subjects

The patients were selected from those referring to the Department of Periodontics, Faculty of Dentistry, Isfahan University of Medical Sciences, during 2014-2015. Twenty patients (10 males and 10 females) with an age range of 20-67 years and a mean age of $36.6 \pm 9.7$ years were selected. The subjects were selected using convenient sampling technique and signed informed consent forms after receiving explanations about the study procedures. The inclusion criteria consisted of age over 18, systemic health, no tobacco use and consent to be included in the study. The exclusion criteria consisted of use of alcohol or tobacco, pregnancy or breastfeeding, use of antimicrobial agents during the previous 2 months and a history of periodontal surgery during the previous year. Finally, a total of 40 sites with primary peri-implantitis in 20 patients were included in the study.

\section{Study Design}

The present double-blind randomized clinical trial had a 3-month follow-up design. Microbial samples were taken from the deepest part of each peri-implant pocket at the beginning of the study and 3 months after treatment. Microbial genome was evaluated using the real-time polymerase chain reaction (RT-PCR) technique. The clinical parameters of probing depth, papilla bleeding index ${ }^{24}$ and modified plaque index (PI ${ }^{25}$ were determined at four points around each implant: distobuccal, mesiobuccal, distolingual and mesiolingual areas.

In the first stage, ultrasonic devices were used to carry out scaling and root planing (SRP) in all the oral cavity areas for every patient (Piezoscaler, Mectron, Carasco GE, Italy). In addition, mechanical debridement was carried out with a carbon fiber curette (Hu-Friedy, Chicago, USA) and air polishing was carried out with a Prophy-Jet (NSK, Tokyo, Japan) and sodium bicarbonate powder around the implants with peri-implantitis. Then all the patients were instructed in oral hygiene, including brushing with modified Bass technique and flossing.

Two weeks after completion of phase I periodontal treatment, the patients were recalled and were divided into test and control groups using a computer randomization table. In the control group, treatment of periodontitis consisted of a combination of mechanical debridement and irradiation with diode laser beams at a wavelength of $810 \mathrm{~nm}$ (Fox, A.R.C. Laser, Gmbh, Germany); in the test group, chemical debridement was combined with PDT by placing the EmunDo photosensitive material (EmunDo, A.R.C. Laser, Gmbh, Germany) within the pocket followed by irradiation with diode laser beams at a wavelength of $810 \mathrm{~nm}$.

\section{Laser Treatment/Photodynamic Therapy}

Treatment was carried out with the use of diode laser beams at a wavelength of $810 \mathrm{~nm}$, using the large-area handpiece and bulb fiber and bare fiber in each area. In the test group, the photosensitizer was injected into the pocket with the use of a direct blunt needle in the apicocoronal direction. After 90 seconds, the photosensitizer was rinsed away with saline solution $(0.9 \% \mathrm{NaOCl})$. The same technique was repeated in the control group with the use of an occluded needle to blind the patients to the procedural steps. Then in both groups, the laser beams were directed toward the pockets using the following steps:

1. Transgingival irradiation: A bleaching handpiece was used for 30 seconds at a laser power of $300 \mathrm{~mW}$ (Figure 1A).

2. Intra-pocket irradiation: A bulb fiber measuring 300 $\mu \mathrm{m}$ in diameter was used to direct laser beams into the pocket with circular movements $(300 \mathrm{~mW}, 30$ seconds) (Figure 1B).

3. Elimination of granulation tissues from the infected pocket: A $300-\mu \mathrm{m}$ bare fiber was used with circular movements (300 $\mathrm{mW}, 30$ seconds) to eliminate the granulation tissues (Figure 1C).

The procedures above were repeated after 2 weeks. All the treatment procedures were carried out by a specialist who was unaware of the principle aims of the study and was 


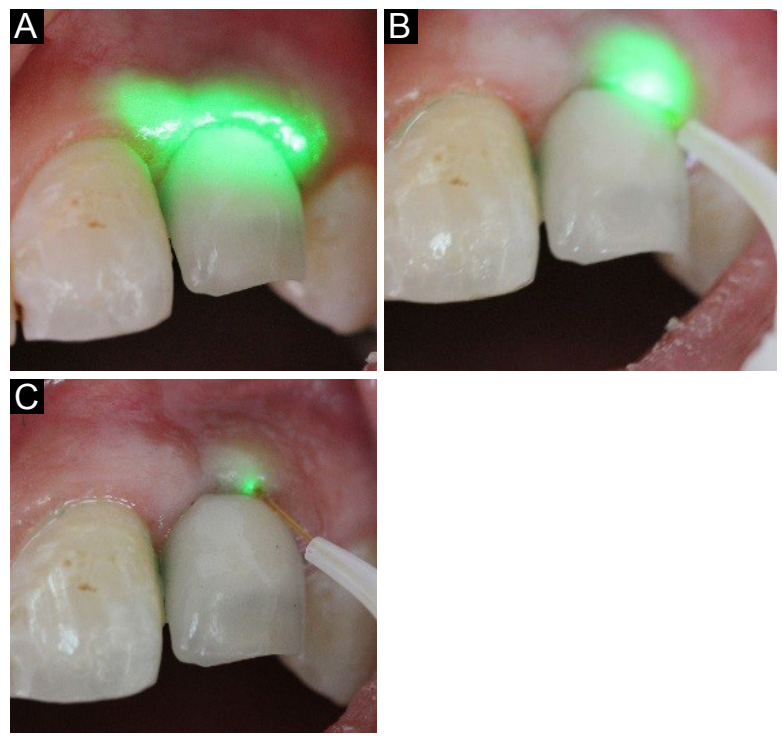

Figure 1. (A) Transgingival Irradiation by Bleaching Handpiece. (B) Intra-pocket Irradiation by a $300 \mu \mathrm{m}$ Bare Fiber in a Circular Pattern. (C) limination of Granulation Tissues From the Infected Pocket Using a $300 \mu \mathrm{m}$ Bare Fiber.

not involved in final evaluations.

During the whole laser irradiation procedures the patients and the personnel wore protective eyeglasses. From the beginning until the end of the study all the patients were examined every two weeks for any complications and to control the plaque and root surface debridement.

\section{Clinical Measurements}

The following clinical parameters were evaluated at baseline and at 6-weeks and 3-months postoperative intervals by a periodontitis who had been confirmed in relation to intra-examiner reproducibility.

Probing pocket depth (PPD): A plastic periodontal probe (Hu-Friedy, Chicago, USA), with a tip diameter of 0.5 $\mathrm{mm}$, was placed with the use of $0.75-\mathrm{N}$ probing force to measure the distance between the gingival margin and the sulcus depth.

PI: PI was determined with the use of modified Mombelli PI (mPI). ${ }^{25}$

Papilla bleeding index (BOP) $)^{24}$ : Papilla PI was evaluated with the use of the same probing force and assessment of bleeding 30 seconds after probing.

\section{Microbiologic Evaluation}

Microbial samples were taken from the deepest part of each pocket at the beginning of the study and 3 months after treatment. A sterile paper point was placed in the pocket depth for 20 seconds and a cotton roll was used for isolation. Then each sample was placed in a sterile vial (pooled sample) and sent to the laboratory for the analysis of the genome. Real-time PCR was used with a conventional kit (Kiagen, USA) for the analysis of microbial samples to determine Aggregatibacter actinomycetemcomitans, Porphyromonas gingivalis,
Prevotella intermedia, Treponema denticola and Tannerella forsythia counts.

\section{Statistical Analysis}

Means and standard deviations of clinical and microbial variables were determined in each group. Data were analyzed with SPSS 20. Kruskal-Wallis test was used to compare non-parametric data (PPD and BOP) between the groups. Mann-Whitney test was used for two-by-two comparison of the groups.

Friedman's and Wilcoxon tests were used to determine differences in mean clinical parameters at baseline and 6-week and 3-month intervals. Wilcoxon test was used to compare microbial variables between the groups at baseline and 3-month postoperative interval $(\alpha=0.05)$.

\section{Results}

Forty implants with primary peri-implantitis in 20 patients were included in the study. All the patients completed the 3-month period of the study. Healing was uneventful in all the cases, without pain, burning sensation or unpleasant feelings. The mean bone loss at the beginning of the study in the laser and PDT groups was $1.02 \pm 0.47$ and $1.45 \pm 0.84 \mathrm{~mm}$, respectively. Independent $t$ test did not reveal any significant difference in the mean bone loss between the two groups $(P=0.56)$.

Table 1 presents the results of clinical parameters at baseline and at 6-week and 3-month postoperative intervals and their changes $(\Delta 0-6 \mathrm{w}, \Delta 0-3 \mathrm{~m})$.

There were no significant differences between the 2 groups at baseline and 6 weeks and 3 months after the therapeutic procedures; however, there was significant decrease in all the clinical parameters after treatment $(P \leq 0.006)$.

Table 2 presents the results of two-by-two comparisons of the clinical parameters during the whole study period. As shown in the table, all the parameters exhibited significant differences at 6-week and 3-month intervals except for the mean of probing depth in the control group and the mean of bleeding index in the test group.

Table 3 presents the counts of periodontopathogens in the cultures at different time intervals and treatment groups. As shown in the table, laser significantly only decreased $P$. gingivalis counts $(P=0.015)$, and differences in $A$. actinomycetemcomitans counts were at significance threshold $(P=0.061)$. On the other hand, laser + EmunDo significantly decreased $A$. actinomycetemcomitans $(P=0.022), \quad T$. forsythia $(P=0.038)$ and $P$. gingivalis $(P=0.050)$ counts. Mann-Whitney test did not reveal any significant difference in changes in bacterial counts before and after treatment between the treatment modalities $\left(P_{A}\right.$. $=0.846, P_{P . \text { gingivalis }}=0.503, P_{P \text {. intermedia }}=0.682$, $\mathrm{P}_{\text {T. denticola }}=0.399$ and $\left.P_{\text {T. forsythia }}=0.199\right)$. Therefore, based on the results, the 2 treatment modalities had similar effects on bacteria.

\section{Discussion}

The results of the present study showed significant decrease in the means of PPD, BOP and $\mathrm{mPI}$ clinical parameters at 
Table 1. The Results of Clinical Parameters at Baseline, 6 Weeks and 3 Months After Treatment And Their Changes ( $\Delta 0-6 \mathrm{w}, \Delta 0-6 \mathrm{~m})$

\begin{tabular}{llccc}
\hline Clinical Parameter & Time Interval & Group Test & Group Control & $\boldsymbol{P}^{\text {Value }}$ \\
\hline & Baseline & $4.06 \pm 0.78$ & $4.02 \pm 0.67$ & 0.872 \\
PPD & 6 Weeks & $2.95 \pm 0.89$ & $2.87 \pm 0.81$ & 0.782 \\
$(\mathrm{~mm})$ & 3 Months & $2.75 \pm 0.84$ & $2.69 \pm 0.77$ & 0.807 \\
& $P$ value (repeated measures ANOVA) & 0.001 & 0.006 & 0.929 \\
$\mathrm{mPI}$ & Baseline & $1.25 \pm 0.64$ & $1.01 \pm 0.91$ & 0.709 \\
Mean score & 6 Weeks & $0.65 \pm 0.49$ & $0.60 \pm 0.75$ & 0.709 \\
& 3 Months & $0.35 \pm 0.49$ & $0.25 \pm 0.44$ & 0.845 \\
BOP & $P$ value (Friedman) & $<0.001$ & $<0.001$ & 0.217 \\
Mean score & Baseline & $1.85 \pm 0.87$ & $2.00 \pm 0.86$ & 0.929 \\
& 6 Weeks & $0.55 \pm 0.69$ & $0.85 \pm 0.67$ & \\
\hline
\end{tabular}

alndependent $t$ test for PPD, Mann-Whitney for $\mathrm{mPI}$ and BOP.

Table 2. The Results of Two-by-Two Comparisons of The Clinical Parameters During the Whole Study Period

\begin{tabular}{|c|c|c|c|c|c|}
\hline \multirow{2}{*}{ Clinical Parameter } & \multirow{2}{*}{ Time Interval } & \multicolumn{2}{|c|}{ Test Group } & \multicolumn{2}{|c|}{ Control Group } \\
\hline & & $\Delta$ & $P$ Value $^{\mathrm{a}}$ & $\Delta$ & $P$ Value $^{\mathrm{a}}$ \\
\hline \multirow{3}{*}{$\begin{array}{l}\text { PPD } \\
(\mathrm{mm})\end{array}$} & $0-6 w$ & $1.11 \pm 1.04$ & $<0.001$ & $1.15 \pm 0.91$ & $<0.001$ \\
\hline & $6 w-3 m$ & $0.20 \pm 0.64$ & 0.039 & $0.19 \pm 0.69$ & 0.087 \\
\hline & $0-3 m$ & $1.31 \pm 1.16$ & $<0.001$ & $1.34 \pm 1.08$ & $<0.001$ \\
\hline \multirow{3}{*}{$\begin{array}{l}\mathrm{mPI} \\
\text { Mean score }\end{array}$} & $0-6 w$ & $1.30 \pm 0.98$ & 0.003 & $1.15 \pm 0.74$ & 0.012 \\
\hline & $6 w-3 m$ & $0.05 \pm 0.60$ & 0.014 & $0.50 \pm 0.51$ & 0.035 \\
\hline & $0-3 \mathrm{~m}$ & $1.35 \pm 0.87$ & $<0.001$ & $1.65 \pm 0.93$ & 0.002 \\
\hline \multirow{3}{*}{$\begin{array}{l}\text { BOP } \\
\text { Mean score }\end{array}$} & $0-6 w$ & $0.60 \pm 0.68$ & $<0.001$ & $0.50 \pm 0.76$ & $<0.001$ \\
\hline & $6 w-3 m$ & $0.30 \pm 0.47$ & 0.705 & $0.35 \pm 0.67$ & 0.002 \\
\hline & $0-3 m$ & $0.90 \pm 0.55$ & $<0.001$ & $0.85 \pm 0.93$ & $<0.001$ \\
\hline
\end{tabular}

a Paired $t$ test for PPD and Wilcoxon for BOP and $\mathrm{mPI}$.

Table 3. The Mean Counts of Periodontopathogens in Microbial Cultures in Terms of Time Intervals and Treatment Groups

\begin{tabular}{|c|c|c|c|c|c|}
\hline Group & Bacteria & Before* & After* & Mean Difference* & $P$ Value (Wilcoxon) \\
\hline \multirow{5}{*}{ Laser } & A. actinomycetemcomitans & $1.12 \pm 0.86$ & $0.61 \pm 0.62$ & $-0.51 \pm 0.85$ & 0.061 \\
\hline & P. gingivalis & $1.68 \pm 1.50$ & $1.03 \pm 1.44$ & $-0.64 \pm 0.90$ & 0.015 \\
\hline & P. intermedia & $1.27 \pm 1.11$ & $0.65 \pm 1.19$ & $-0.62 \pm 1.35$ & 0.091 \\
\hline & T. denticola & $0.48 \pm 0.55$ & $0.28 \pm 0.44$ & $-0.20 \pm 0.46$ & 0.26 \\
\hline & T. forsythia & $0.31 \pm 0.55$ & $0.15 \pm 0.27$ & $-0.16 \pm 0.57$ & 0.481 \\
\hline \multirow{5}{*}{ Laser + EmunDo } & A. actinomycetemcomitans & $0.91 \pm 0.80$ & $0.47 \pm 0.64$ & $0.64 \pm 0.44$ & 0.022 \\
\hline & P. gingivalis & $1.42 \pm 1.49$ & $0.70 \pm 0.99$ & $1.49 \pm 0.72$ & 0.050 \\
\hline & P. intermedia & $1.04 \pm 1.30$ & $0.39 \pm 0.58$ & $1.59 \pm 0.65$ & 0.182 \\
\hline & T. denticola & $0.53 \pm 0.63$ & $0.21 \pm 0.46$ & $0.71 \pm 0.32$ & 0.085 \\
\hline & T. forsythia & $0.43 \pm 0.55$ & $0.14 \pm 0.24$ & $0.55 \pm 0.29$ & 0.038 \\
\hline
\end{tabular}

6-week and 3-month postoperative intervals compared to baseline in both groups; however, there were no significant difference between the two groups. In relation to BOP, PDT did not result in a significant difference after 3 months compared to the 6-week interval; however, such a difference was significant in the laser group. Therefore, there was no significant change after 3 months compared to 6-week interval. However, contrary to BOP, PPD exhibited greater decrease in the laser group; in this context, after 3 months no significant difference was observed compared to the 6-week interval. In addition, there were significant differences in PI between the three time intervals in both groups. It shows that during the study period, the oral hygiene of patients has improved.
On the other hand, the results of the present study showed no significant difference in decreasing bacterial counts between the two treatment modalities; the two techniques decreased all the bacterial counts, but eliminated none. Therefore, it can be concluded that both treatment modalities were effective in decreasing the counts of periodontopathogens. However, during the follow-ups in the present study, the differences were significant in the laser group only in relation to $P$. gingivalis; the differences were at significance threshold in relation to $A$. actinomycetemcomitans. In addition, in the PDT group, A. actinomycetemcomitans, $P$. gingivalis and T. forsythia counts decreased significantly.

Salvi et $\mathrm{al}^{26}$ showed that mechanical debridement alone 
around implants with carbon fiber curettes resulted in a decrease in inflammation severity in the mucosa and a decrease in pocket depth. However, many other studies, including that of Birang et al, ${ }^{27}$ have shown that the use of diode laser or chlorhexidine gel, as adjunctive techniques, is more effective than mechanical debridement alone in the treatment of chronic periodontitis, concluding that if routine mechanical debridement is combined with diode laser or chlorhexidine gel, it will be more effective in improving the clinical and microbiologic parameters. Gojkov-Vakelic et $\mathrm{a}^{28}$ showed that use of diode laser beams decreased the number of active pathogens in periodontal pockets. On the other hand, the results of studies done by Moritz et $\mathrm{al}^{29}$ and Angelov et $\mathrm{al}^{30}$ showed that treatment with diode laser might improve periodontal pockets and periodontal parameters. In a case report, Roncati et $\mathrm{al}^{1}$ showed that the use of diode laser resulted in a decrease in probing depth and in a negative BOP around implants. Therefore, given the results of previous studies in relation to the effect of diode laser on decreasing periodontal pathogens and similarity between the microbial agents in periodontitis and peri-implantitis, in the present study, diode laser with a wavelength of 810 $\mathrm{nm}$ was used to improve peri-implantitis parameters. In addition, considering reports on the light absorption and florescence properties of different dyes, it was shown that excitation of these materials with light results in destructive effects in biologic systems. ${ }^{31}$

Von Tappeiner ${ }^{32}$ showed that these reactions can result in the destruction of protozoa. Blum ${ }^{33}$ and Spikes and Livingston $^{34}$ believed that PDT function depended on activated photoreactions in which the oxygen molecule takes part, resulting in photosensitized dye oxidation.

There is controversy over the effect of PDT. The majority of reports indicate that PDT only results in decreasing the inflammation severity and there is insufficient evidence in relation to the effect of PDT on periodontitis. ${ }^{35-37}$ Shibli et $\mathrm{al}^{38}$ showed the effect of PDT on the pathogens within periodontal pockets. Meisel and Kocher ${ }^{20}$ showed that the use of photosensitizing dyes and their activation with laser beams might result in the destruction of pathogens responsible for periodontal diseases and peri-implantitis. Gursoy et $\mathrm{al}^{39}$ suggested that PDT might be an appropriate tool in superficial and local infections, and although it cannot be an alternative for antimicrobial agents, it might facilitate the treatment of oral infections. Therefore, considering the results of studies on the effect of PDT on periodontal pathogens, periodontal infections and periimplantitis, in the present study PDT was applied in the test group and its effects were compared with those of laser therapy (LT) alone in the control group.

Schar et $\mathrm{al}^{2}$ compared the effects of PDT and during therapy with minocycline on peri-implantitis. The results showed that both treatment modalities were similarly effective in decreasing inflammation of the peri-implant mucosa.

Bassetti et $\mathrm{al}^{40}$ compared drug therapy with minocycline and PDT for the treatment of peri-implantitis. A follow- up of 12 months showed a significant decrease in the number of sites with BOP, a decrease in probing depth and a decrease in $P$. gingivalis and T. forsythia counts, with no significant difference between the 2 groups.

The results of the present study in relation to a decrease in mucosal inflammation, a decrease in BOP and PPD and a decrease in bacterial colony counts are consistent with those of studies by Schar et $\mathrm{al}^{2}$ and Bassetti et al. ${ }^{40}$ Therefore, it can be concluded that treatment with laser, drug therapy and treatment with laser in association with photosensitizing dyes yield similar results in the treatment of peri-implantitis. On the other hand, based on the results of the present study only $P$. gingivalis counts in the control group and $A$. actinomycetemcomitans, $P$. gingivalis and $T$. forsythia counts in the test group decreased significantly; in this context, the bacterial species were similar to those in the study carried out by Bassetti et al. ${ }^{40}$

In a study, the effect of PDT with toluidine blue on periodontopathogens and biofilm on tooth surfaces was evaluated and it was reported that the use of diode laser beams at a wavelength of $830 \mathrm{~nm}$ in association with the use of toluidine blue within periodontal pockets was effective in destroying bacteria and it might be a reliable alternative for antimicrobial treatment in periodontitis. ${ }^{41}$ Therefore, the results of the present study are consistent with studies carried on by Shibli et al, ${ }^{38}$ Kocher and Mecisel $^{20}$ and the study mentioned above. PDT with a combination of laser and EmunDo dye resulted in destruction of bacteria and in a decrease in bacterial counts, indicating that the type of the dye does not influence the antibacterial effect and the presence of a different photosensitizing dye results in similar antibacterial effects. On the other hand, there was no significant difference between the use of laser beams alone and in association with a photosensitizing agent in the present study. Therefore, it can be concluded that dye does not have a major role in the antimicrobial effect of laser and laser alone is responsible for the antibacterial effect.

One of the limitations of the present study was the absence of comparisons between mechanical treatment alone and the two modalities of treatment with laser beams. In addition, another limitation was the lack of comparison of the results with the drug therapy technique. It is suggested that a similar study be carried out with a larger sample size in order to compare the effects of mechanical treatment, drug therapy and the use of laser beams.

\section{Conclusion}

Based on the results of this study, 810-nm laser beams alone and in association with photo-sensitizing dye resulted in improvements in the clinical and microbiological parameters around implants with peri-implantitis during the short follow-up periods of the study, with no significant difference between the 2 groups.

\section{Ethical Considerations}

The present randomized clinical trial study was registered at the Iranian Clinical Trials website under the code 
IRCT2017030932749N2 (http://irct.ir) and was approved by the Ethics Committee of Isfahan University of Medical Sciences under the code 394383.

\section{Conflict of Interests}

None.

\section{References}

1. Roncati M, Lucchese A, Carinci F. Non-surgical treatment of peri-implantitis with the adjunctive use of an 810-nm diode laser. J Indian Soc Periodontol. 2013;17(6):812-815. doi: 10.4103/0972-124X.124531.

2. Schar D, Ramseier CA, Eick S, Arweiler NB, Sculean A, Salvi GE. Anti-infective therapy of peri-implantitis with adjunctive local drug delivery or photodynamic therapy: six-month outcomes of a prospective randomized clinical trial. Clin Oral Implants Res. 2013;24(1):104-110. doi: 10.1111/j.1600-0501.2012.02494.x.

3. Novaes AB Jr, Schwartz-Filho HO, de Oliveira RR, Feres M, Sato S, Figueiredo LC. Antimicrobial photodynamic therapy in the non-surgical treatment of aggressive periodontitis: microbiological profile. Lasers Med Sci. 2012;27(2):389-395. doi: 10.1007/s10103-011-0901-6.

4. Feres M, Bernal M, Matarazzo F, Faveri M, Duarte PM, Figueiredo LC. Subgingival bacterial recolonization after scaling and root planing in smokers with chronic periodontitis. Aust Dent J. 2015;60(2):225-232. doi: 10.1111/adj.12225.

5. Zhu C, Yang J, Sun J, Shi J, Gou J, Li A. Induction of immune response and prevention of alveolar bone loss with recombinant Porphyromonas gingivalis peptidylarginine deiminase. Arch Oral Biol. 2013;58(12):1777-1783. doi: 10.1016/j.archoralbio.2013.09.006.

6. Feres M, Soares GMS, Mendes JAV, et al. Metronidazole alone or with amoxicillin as adjuncts to non-surgical treatment of chronic periodontitis: a 1-year doubleblinded, placebo-controlled, randomized clinical trial. $J$ Clin Periodontol. 2012;39(12):1149-1158.

7. Krishna MK, Ravindran SK, Vivekanandan G, Navasivayam A, Thiagarajan R, Mohan R. Effects of a single episode of subgingival irrigation with tetracycline $\mathrm{HCl}$ or chlorhexidine: a clinical and microbiological study. J Indian Soc Periodontol. 2011;15(3):245.

8. Sperandio FF, Huang YY, Hamblin MR. Antimicrobial photodynamic therapy to kill gram-negative bacteria. Recent Pat Antiinfect Drug Discov. 2013;8(2):108-120.

9. Ethirajan M, Chen Y, Joshi P, Pandey RK. The role of porphyrin chemistry in tumor imaging and photodynamic therapy. Chem Soc Rev. 2011;40(1):340-362. doi: 10.1039/ b915149b.

10. Cassidy CM, Donnelly RF, Elborn JS, Magee ND, Tunney MM. Photodynamic Antimicrobial Chemotherapy (PACT) in combination with antibiotics for treatment of Burkholderia cepacia complex infection. J Photochem Photobiol B. 2012;106:95-100. doi: 10.1016/j. jphotobiol.2011.10.010.

11. Mang TS, Tayal DP, Baier R. Photodynamic therapy as an alternative treatment for disinfection of bacteria in oral biofilms. Lasers Surg Med. 2012;44(7):588-596. doi: 10.1002/lsm.22050.

12. Takasaki AA, Aoki A, Mizutani K, Schwarz F, Sculean A, Wang CY, et al. Application of antimicrobial photodynamic therapy in periodontal and peri-implant diseases. Periodontol 2000. 2009;51:109-40. doi: 10.1111/j.16000757.2009.00302.x.

13. Dortbudak O, Haas R, Bernhart T, Mailath-Pokorny G. Lethal photosensitization for decontamination of implant surfaces in the treatment of peri-implantitis. Clin Oral Implants Res. 2001;12(2):104-108.

14. Haas R, Baron M, Dortbudak O, Watzek G. Lethal photosensitization, autogenous bone, and e-PTFE membrane for the treatment of peri-implantitis: preliminary results. Int $J$ Oral Maxillofac Implants. 2000;15(3):374-382.

15. Garcez AS, Ribeiro MS, Tegos GP, Nunez SC, Jorge AO, Hamblin MR. Antimicrobial photodynamic therapy combined with conventional endodontic treatment to eliminate root canal biofilm infection. Lasers Surg Med. 2007;39(1):59-66.

16. Bagchi B, Basu S. Role of dye molecules remaining outside the cell during photodynamic inactivation of Escherichia coli in the presence of acriflavine. Photochem Photobiol. 1979;29(2):403-405.

17. Theodoro LH, Silva SP, Pires JR, et al. Clinical and microbiological effects of photodynamic therapy associated with nonsurgical periodontal treatment. A 6-month follow-up. Lasers Med Sci. 2012;27(4):687-693. doi: 10.1007/s10103-011-0942-x.

18. Ehrenberg B, Gross E, Nitzan Y, Malik Z. Electric depolarization of photosensitized cells: lipid vs. protein alterations. Biochim Biophys Acta. 1993;1151(2):257-264.

19. Pereira Gonzales F, Maisch T. Photodynamic inactivation for controlling Candida albicans infections. Fungal Biol. 2012;116(1):1-10.

20. Meisel P, Kocher T. Photodynamic therapy for periodontal diseases: state of the art. J Photochem Photobiol B. 2005;13;79(2):159-170.

21. Kreisler M, Al Haj H, d'Hoedt B. Clinical efficacy of semiconductor laser application as an adjunct to conventional scaling and root planing. Lasers Surg Med. 2005;37:350-355.

22. Romanos GE, Henze M, Banihashemi S, Parsanejad HR, Winckler J, Nentwig GH. Removal of epithelium in periodontal pockets following diode $(980 \mathrm{~nm})$ laser application in the animal model: an in vitro study. Photomed Laser Surg. 2004;22(3):177-183.

23. Cobb CM. Lasers in periodontics: a review of the literature. J Periodontol. 2006;77(4):545-564.

24. Saxer U, Mühlemann H. Motivation and education (German). SSO Schweiz Monatsschr Zahnheilkd. 1975;85(9):905-919.

25. Mombelli A, Oosten M, Schürch E, Lang N. The microbiota associated with successful or failing osseointegrated titanium implants. Oral Microbiol Immunol. 1987;2(4):145151.

26. Salvi GE, Persson GR, Heitz-Mayfield LJ, Frei M, Lang NP. Adjunctive local antibiotic therapy in the treatment of peri-implantitis II: clinical and radiographic outcomes. Clin Oral Implants Res. 2007;18(3):281-285.

27. Birang R, Yaghini J, Adibrad M, Kiany S, Mohammadi Z, Birang $\mathrm{E}$. The effects of diode laser and chlorhexidine gel in the treatment of chronic periodontitis. J Lasers Med Sci. 2011;2(4):131-8

28. Gojkov-Vukelic M, Hadzic S, Dedic A, Konjhodzic R, Beslagic E. Application of a diode laser in the reduction 
of targeted periodontal pathogens. Acta Inform Med. 2013;21(4):237-240. doi: 10.5455/aim.2013.21.237-240.

29. Moritz A, Schoop U, Goharkhay K, et al. Treatment of periodontal pockets with a diode laser. Lasers Surg Med. 1998;22:302-311.

30. Angelov N, Pesevska S, Nakova M, et al. Periodontal treatment with a low-level diode laser: clinical findings. Gen Dent. 2009;57(5):510-513.

31. Marcacci A. Sur l'action des alcaloides dans le regne vegetal et animal. Arch Ital Biol. 1888;9:2-4.

32. Von Tappeiner H. Uber die Wirkung fluoreszierender Stoffe auf Infusorien nach Versuchen von O. Raab. Muench Med Wochenschr. 1900;47:5.

33. Blum HF. Photodynamic action and diseases caused by light. J Nerv Ment Dis. 1943;98(3):332.

34. Spikes JD, Livingston R. The molecular biology of photodynamic action: sensitized photo-autooxidations in biological systems. In: Augenstein L, Mason R, Zelle M, eds. Advances in Radiation Biology. New York: Academic Press; 1967:29-121.

35. Andersen R, Loebel N, Hammond D, Wilson M. Treatment of periodontal disease by photodisinfection compared to scaling and root planing. J Clin Dent. 2007;18(2):34-38.

36. Braun A, Dehn C, Krause F, Jepsen S. Short-term clinical effects of adjunctive antimicrobial photodynamic therapy in periodontal treatment: a randomized clinical trial. J Clin Periodontol. 2008;35(10):877-884.

37. Azarpazhooh A, Shah PS, Tenenbaum HC, Goldberg MB. The effect of photodynamic therapy for periodontitis: a systematic review and meta-analysis. $J$ Periodontol. 2010;81(1):4-14. doi: 10.1902/jop.2009.090285.

38. Shibli JA, Martins MC, Theodoro LH, Lotufo RF, Garcia VG, Marcantonio EJ. Lethal photosensitization in microbiological treatment of ligature-induced periimplantitis: a preliminary study in dogs. J Oral Sci. 2003;45(1):17-23. doi: 10.1007/s00784-012-0845-7.

39. Gursoy H, Ozcakir-Tomruk C, Tanalp J, Yilmaz S. Photodynamic therapy in dentistry: a literature review. Clin Oral Investig. 2013;17(4):1113-1125.

40. Bassetti M, Schar D, Wicki B, et al. Anti-infective therapy of peri-implantitis with adjunctive local drug delivery or photodynamic therapy: 12-month outcomes of a randomized controlled clinical trial. Clin Oral Implants Res. 2014;25(3):279-287. doi: 10.1111/clr.12155.

41. Traini T, Degidi M, Iezzi G, Artese L, Piattelli A. Comparative evaluation of the peri-implant bone tissue mineral density around unloaded titanium dental implants. J dent. 2007;35(1):84-92. 